\title{
Delivery of continuously-varying stimuli using channelrhodopsin-2
}

\author{
Tatjana Tchumatchenko ${ }^{1 * t}$, Jonathan P. Newman ${ }^{2 * t}$, Ming-fai Fong ${ }^{2,3}$ and Steve M. Potter \\ ${ }^{1}$ Max Planck Institute for Brain Research, Frankfurt am Main, Germany \\ 2 Department of Biomedical Engineering, Georgia Institute of Technology, Atlanta, GA, USA \\ ${ }^{3}$ Department of Physiology, Emory University School of Medicine, Atlanta, GA, USA
}

Edited by:

Hollis Cline, The Scripps Research Institute, USA

\section{Reviewed by:}

Michael A. Lane, University of

Florida, USA

Michael Nitabach, Yale University

School of Medicine, USA

\section{*Correspondence:}

Dr. Tatjana Tchumatchenko, Max

Planck Institute for Brain Research,

Max-von-Laue-Str. 4, 60438

Frankfurt am Main, Germany

e-mail: tatjana.tchumatchenko@

brain.mpg.de

Dr. Jonathan P. Newman,

Department of Biomedical

Engineering, Georgia Institute of

Technology, 313 Ferst Drive, Atlanta,

USA

e-mail: jnewman6@gatech.edu

${ }^{\dagger}$ Co-first authors; These authors

have contributed equally to this work.
To study sensory processing, stimuli are delivered to the sensory organs of animals and evoked neural activity is recorded downstream. However, noise and uncontrolled modulatory input can interfere with repeatable delivery of sensory stimuli to higher brain regions. Here we show how channelrhodopsin-2 (ChR2) can be used to deliver continuous, subthreshold, time-varying currents to neurons at any point along the sensory-motor pathway. To do this, we first deduce the frequency response function of ChR2 using a Markov model of channel kinetics. We then confirm ChR2's frequency response characteristics using continuously-varying optical stimulation of neurons that express one of three ChR2 variants. We find that wild-type ChR2 and the E123T/H134R mutant ("ChETA") can pass continuously-varying subthreshold stimuli with frequencies up to $\sim 70 \mathrm{~Hz}$. Additionally, we find that wild-type ChR2 exhibits a strong resonance at $\sim 6-10 \mathrm{~Hz}$. Together, these results indicate that ChR2-derived optogenetic tools are useful for delivering highly repeatable artificial stimuli that mimic in vivo synaptic bombardment.

Keywords: channelrhodopsin-2, linear response theory, dynamical systems, neural circuits, networks and dynamical systems, circuit dynamics, optogenetics, electrophysiology methods

\section{INTRODUCTION}

The network response to continuously-varying stimuli is at the core of cognitive and sensory processing. To understand how neuronal networks encode and process continuously-varying input, a sensory organ is presented with a precisely-defined stimulus and evoked spiking activity is recorded from a corresponding brain region. The power of this technique for deducing network encoding properties has been demonstrated in numerous preparations, including the retina, (Warland et al., 1997; Chichilnisky, 2001), antennal and mechanosensory systems of insects (Warland et al., 1992; Geffen et al., 2009), and somatosenory, auditory, and visual systems of mammals (Arabzadeh et al., 2003; Lesica et al., 2007; Kayser et al., 2010). However, as stimuli delivered to sensory organs propagate to higher brain areas, intrinsic noise and modulatory input from secondary brain regions can interfere with controlled input signals. For studies concerning the function of neural circuits that are several synapses removed from sensory input, the direct introduction of continuously-varying currents to a neural population may provide a more straightforward way to deduce circuit response dynamics.

Optogenetic methods allow precise control of spike times using brief light pulses to excite light-gated ion channels and pumps, such as channelrhodopsin-2 (ChR2) (Boyden et al., 2005; Gunaydin et al., 2010; Mattis et al., 2011). Pulsed optical stimulation using ChR2 dictates a spiking response that is tightly locked to each stimulus by briefly overriding neuronal dynamics. This stands in contrast to the highly variable, sub-threshold currents recorded from cortical neurons during natural sensory processing in vivo (Destexhe et al., 2003). We hypothesized that using relatively low intensity, continuously modulated optical stimuli to excite ChR2 might allow conductance fluctuations that mimic in vivo-like synaptic bombardment and leave the decision of when and how often to spike to individual cells (Mainen and Sejnowski, 1995; Tchumatchenko et al., 2011). Surprisingly, while the response properties of microbial opsins to optical pulses have been studied extensively (Mattis et al., 2011), little is known about their ability to relay fluctuating light signals.

In order for ChR2 to be useful for delivering continuouslyvarying currents, it must allow (1) sufficient bandwidth to mimic synaptic communication and (2) repeatable current waveforms to be delivered across trials. Here, we address these requirements theoretically and experimentally. We find that wild-type ChR2 (ChR2) (Boyden et al., 2005) supports significant photocurrents up to $69 \mathrm{~Hz}$, the $\mathrm{H} 134 \mathrm{R}$ mutant $\left(\mathrm{ChR} 2_{\mathrm{R}}\right.$ ) (Nagel et al., 2005) up to $37 \mathrm{~Hz}$, and E123T/H134R mutant $\left(\mathrm{ChR} 2_{\mathrm{A}}\right.$; also known as "ChETA") (Gunaydin et al., 2010) up to $73 \mathrm{~Hz}$, and show that evoked current waveforms are extremely repeatable across trials. Using the model, we find that the bandwidth over which $\mathrm{ChR} 2 \mathrm{R}$ and $\mathrm{ChR} 2$ can convey time-varying stimuli is reduced with increasing membrane potential but that $\mathrm{ChR} 2{ }_{\mathrm{A}}$ 's passband is unaffected. Finally, we show that wild type ChR2 supports a strong resonance with a natural frequency around $10 \mathrm{~Hz}$. This resonance is present, but significantly attenuated, in the H134R and E123T/H134R mutants. 


\section{RESULTS}

\section{ChR2's FREQUENCY RESPONSE}

In this study, we sought a general description of ChR2's dynamics that captured the ability of both ChR2 and its engineered variants to convey continuously-varying stimuli. To do this, we determined the frequency response function of a population of channels expressed by a single cell, $F_{\mathrm{ChR} 2}(\omega)$, using a three-state Markov model of ChR2's channel kinetics (Figure 1A) (Nagel et al., 2003). The rate equations governing the model's state transitions are

$$
\begin{aligned}
& \dot{O}(t)=\epsilon \phi(t) C(t)-\Gamma_{d} O(t) \\
& \dot{D}(t)=\Gamma_{d} O(t)-\Gamma_{r} D(t) \\
& C(t)=1-O(t)-D(t),
\end{aligned}
$$

where the state variables $O, D$, and $C$ are the probabilities of a channel being open, desensitized, or closed, respectively. $\Gamma_{d}$ and $\Gamma_{r}$ are the rates of channel desensitization and recovery. $\epsilon$ is the quantum efficiency of ChR2 and $\phi(t)$ is the instantaneous photon flux (light intensity) impinging on a single channel. $\phi(t)$ can be modulated by changing the light intensity of a stimulating light source as a function of time.

The conductance of ChR2 across the cell membrane is proportional to the number of channels that occupy the open state. Therefore, $F_{\mathrm{ChR} 2}(\omega)$ can be thought of as a frequency- and phasedependent transition rate from the channels' closed to open states in response to a continuously-varying stimulus. Since individual channels switch between states discretely, $F_{\mathrm{ChR} 2}(\omega)$ describes the transformation of arbitrary optical waveforms to intracellular current under the assumption that a large number of channels are present in the cell's membrane. $F_{\mathrm{ChR} 2}(\omega)$ is given by

$F_{\mathrm{ChR} 2}(\omega)=\frac{C_{0}\left(j \omega+\Gamma_{r}\right)}{-\omega^{2}+j \omega\left(\Gamma_{r}+\epsilon \phi_{0}+\Gamma_{d}\right)+\epsilon \phi_{0} \Gamma_{r}+\epsilon \phi_{0} \Gamma_{d}+\Gamma_{r} \Gamma_{d}}$.
A detailed derivation of $F_{\mathrm{ChR} 2}(\omega)$ can be found in the Methods section.

The amplitude response function, $\left|F_{\mathrm{ChR2}}(\omega)\right|$ (Equations 4 and 19), is the component of $F_{\mathrm{ChR2}}(\omega)$ that indicates the frequency-dependent gain of the channel population in response to fluctuating light signals. The predicted amplitude response functions for $\operatorname{ChR} 2,\left|F_{\mathrm{ChR} 2}(\omega)\right|, \quad \mathrm{ChR} 2_{\mathrm{R}},\left|F_{\mathrm{ChR} 2 \mathrm{R}}(\omega)\right|$, and $\mathrm{ChR} 2_{\mathrm{A}},\left|F_{\mathrm{ChR2}_{\mathrm{A}}}(\omega)\right|$, are shown in Figure 1B for different mean illumination intensities. Model parameters were obtained for each ChR2 variant by fitting the predicted amplitude response function (Equation 19) to the experimental estimate (Table 1; Methods section "Delivering time varying currents using ChR2").

$\left|F_{\mathrm{ChR} 2}(\omega)\right|$ has a high frequency cutoff (width at half maximum) of $69 \mathrm{~Hz}$. It should be noted that this cutoff value is defined relative to ChR2's peak conductance, and not in terms of absolute photocurrents. For this reason, it is still possible to use ChR2 to deliver physiologically significant photocurrents at stimulation frequencies exceeding $69 \mathrm{~Hz}$. The shape of $\left|F_{\mathrm{ChR} 2}(\omega)\right|$ indicates that $\mathrm{ChR} 2$ exhibits a significant resonance with a natural frequency around $6-10 \mathrm{~Hz}$. This feature explains the large peak to steadystate (DC) ratio of ChR2-mediated photocurrents in response to pulsed stimuli (Gunaydin et al., 2010; Mattis et al., 2011).

In agreement with previous characterizations, $\mathrm{ChR} 2 \mathrm{R}$ is significantly slower than $\mathrm{ChR} 2$ and $\left|F_{\mathrm{ChR} 2 \mathrm{R}}(\omega)\right|$ has a cutoff frequency at $37 \mathrm{~Hz}$ (Mattis et al., 2011). While $\mathrm{ChR} 2 \mathrm{R}$ supports a resonance in the $3-4 \mathrm{~Hz}$ range, its effect is significantly reduced compared

Table 1 | Markov model parameters for each ChR2 variant obtained from fitting the amplitude response function (Equation19) to the empirically derived response function.

\begin{tabular}{llll}
\hline Variant & $\boldsymbol{\epsilon \phi}_{\mathbf{0}}\left(\mathbf{s}^{\mathbf{- 1}}\right)$ & $\Gamma_{\boldsymbol{d}}\left(\mathbf{s}^{\mathbf{- 1}}\right)$ & $\Gamma_{\boldsymbol{r}}\left(\mathbf{s}^{\mathbf{- 1}}\right)$ \\
\hline ChR2 & 6.51 & 236.35 & 3.60 \\
ChR2 $_{\mathrm{R}}$ & 1.16 & 126.74 & 8.38 \\
ChR2 $_{A}$ & 0.96 & 254.63 & 5.57 \\
\hline
\end{tabular}

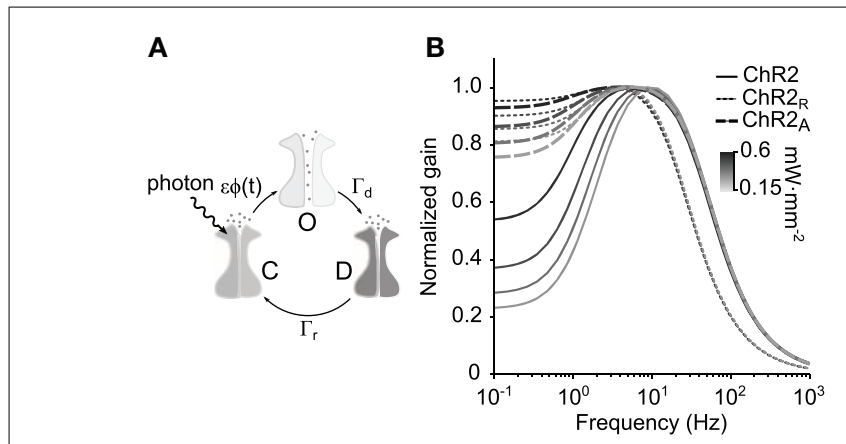

FIGURE 1 | ChR2's amplitude response function. (A) Illustration of the three-state Markov channel model described by Equations 1-3. The transition rates between open, $O$, desensitized, $D$, and closed, $C$, states are $\epsilon \phi(t), \Gamma_{r}$, and $\Gamma_{d}$, respectively. (B) Amplitude response functions $\left|F_{\mathrm{ChR} 2}(2 \pi f)\right|$ for the model are shown for three ChR2 variants using different mean illumination intensities $\left(0.15-0.6 \mathrm{~mW} \cdot \mathrm{mm}^{-2}\right)$ and parameters in Table 1. (C) Voltage dependence of ChR2's amplitude
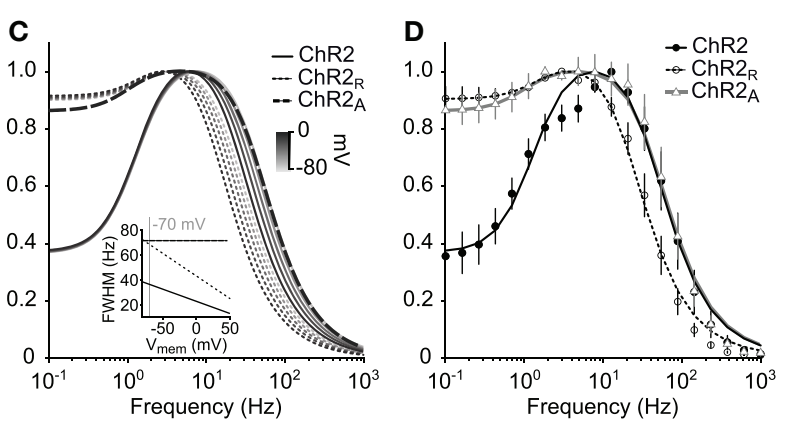

response function. $\mathrm{ChR} 2$ and $\mathrm{ChR} 2_{\mathrm{R}}$ both have a voltage-dependent desensitization rate, $\Gamma_{d}(v)$, which results in decreased bandwidth as the membrane potential increases. $\mathrm{ChR} 2_{\mathrm{A}}$ does not have a voltage dependent desensitization rate and therefore has a stable bandwidth across membrane potentials. (D) Predicted amplitude response of each ChR2 variant compared to the experimentally measured response for a mean illumination intensity of $0.35 \mathrm{~mW} \cdot \mathrm{mm}^{-2}$. Error bars are \pm 1 STD. 
to ChR2. The bandwidth of $\mathrm{ChR} 2 \mathrm{~A}$ was similar to that of ChR2 with a high frequency cutoff of $73 \mathrm{~Hz} .\left|F_{\mathrm{ChR} 2_{\mathrm{A}}}(\omega)\right|$ displayed a moderate resonance that peaked at $3-5 \mathrm{~Hz}$ depending on the mean light intensity of the stimulation waveform.

\section{VOLTAGE DEPENDENCE OF CHANNEL KINETICS}

For some ChR2 variants, channel kinetics are dependent on the membrane potential. The off time of ChR2- and ChR2 $\mathrm{R}^{-}$ evoked currents grow with increasing membrane potentials (Mattis et al., 2011). Additionally, the time-to-peak conductance for ChR2 increases approximately linearly with membrane potential (Berndt et al., 2011; Mattis et al., 2011). ChR2 2 does not have voltage dependent kinetics (Berndt et al., 2011; Mattis et al., 2011). Therefore, for the ChR2 and ChR2 $\mathrm{R}$ variants, the transition rates $\Gamma_{r}$ and $\Gamma_{d}$ in the Markov model (Equations 1-3) both could be voltage dependent. To understand how voltage-dependent kinetics affect the bandwidth of each ChR2 variant, we derived the transient response of our model to a delta pulse of magnitude $\phi_{0}$ and to a downward step to zero from initial intensity $\phi_{0}$. The response to a delta light pulse, ("on-dynamics") is given by

$$
O^{\text {on }}(t)=\epsilon \phi_{0} \exp \left(-\Gamma_{d} t\right) \theta(t)
$$

where $\theta(t)$ is the Heaviside theta function. The response to a downward step ("off-dynamics") is given by

$$
O^{\text {off }}(t)=\exp \left(-\Gamma_{d} t\right) \theta(t) \frac{\Gamma_{r} \epsilon \phi_{0}}{\Gamma_{d} \Gamma_{r}+\Gamma_{d} \epsilon \phi_{0}+\Gamma_{r} \epsilon \phi_{0}} .
$$

The long time dynamics of both $O^{\text {on }}$ and $O^{\text {off }}$ are dominated by $\Gamma_{d}$ when using biophysically relevant parameters. Therefore, to capture the effect of voltage on channel kinetics, we assumed a linear relationship between the voltage and $\Gamma_{d}$ according to $\Gamma_{d}(v)=\Gamma_{d}(1-0.0056(v+70 \mathrm{mV}))$ (Mattis et al., 2011). We then recalculated the amplitude response function at membrane potentials ranging from -80 to $0 \mathrm{mV}$ (Figure 1C). Increases in membrane potential affected the high-frequency cutoff for the $\mathrm{ChR} 2$ and $\mathrm{ChR} 2_{\mathrm{R}}$ and had a large effect on channel bandwidth. As the membrane voltage increased from -80 to $0 \mathrm{mV}$, the bandwidth of the amplitude response function decreased by $37 \%$ for both variants (Figure 1C, inset). In contrast, ChR2 A's constant bandwidth across voltages makes it well suited for introducing continuously-varying conductances into cells that are not voltage-clamped.

\section{ROBUSTNESS OF THE FREOUENCY RESPONSE FUNCTION}

The linear time-invariant frequency response function has the greatest predictive power for stimuli with low peak-to-peak amplitudes. To test the robustness of the frequency response function when using larger amplitude inputs, we compared it with the complete response arising from time-varying light amplitude. Sinusoidal inputs with mean intensity of $\phi_{0}=0.35 \mathrm{~mW} \cdot \mathrm{mm}^{-2}$ and different amplitudes $\delta \phi$ were used to drive the linear timeinvariant frequency response (Equation 4) according to

$$
O(t)=O_{0}+\epsilon \delta \phi F_{\mathrm{ChR} 2}(\omega) \exp (j \omega t)
$$

The complete response dynamics of the Markov model were obtained by inserting the offset and amplitude parameters, $\phi_{0}$ and
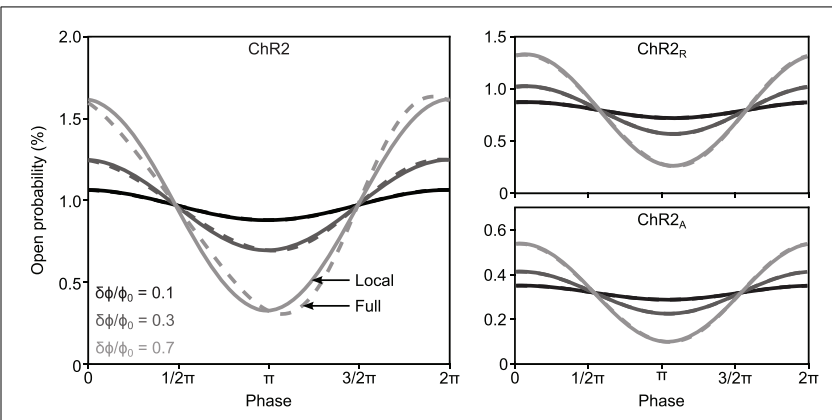

FIGURE 2 | $F_{\text {ChR2 }}(\omega)$ 's linear time-invariant response versus complete model dynamics for different $\mathbf{C h R 2}$ variants. The time-invariant $F_{\mathrm{ChR} 2}(\omega)$ approximation (solid lines) and the complete dynamics (dashed lines) of $O(t)$ are shown in response to $5 \mathrm{~Hz}$ sinusoidal stimuli for ChR2 (left), ChR2 $\mathrm{R}$ (right, top) and $\mathrm{ChR}_{2}$ (right, bottom). Gray shades represent different sinusoidal amplitudes normalized to the mean stimulus intensity, $\delta \phi / \phi_{0}=0.1,0.3$, and 0.7 .

$\delta \phi$, into the sinusoidal drive (Equation 8 ) and numerically integrating the corresponding differential Equations (1-3) to obtain $O(t)$.

The response predicted by $F_{\mathrm{ChR} 2}(\omega)$ and complete solutions will match when $\left|i \omega+\epsilon \phi_{0}\right| \gg \epsilon \delta \phi$. This condition guarantees that terms proportional to $\exp (j \omega t)$ dominate over higher order terms in the derivation of the $F_{\mathrm{ChR} 2}(\omega)$ response function (Equations 13, 14). This condition is fulfilled when $\delta \phi \ll \phi_{0}$ or for frequencies $\omega \gg \epsilon \phi_{0}$. We compared simulated and timeinvariant solutions to Equations 1-3 and found that the frequency response function provided a good approximation of the complete dynamics, even for relatively large stimulus amplitudes and low stimulus frequencies, where deviations between the linear time-invariant response and complete model dynamics are largest ( $5 \mathrm{~Hz}$ input; Figure 2). The deviation between $F_{\mathrm{ChR} 2}(\omega)$ and complete dynamics was negligible for higher stimulus frequencies (20 Hz input; Supplementary Figure S4).

\section{DELIVERING CONTINUOUSLY-VARYING CURRENTS USING ChR2}

To verify $F_{\mathrm{ChR} 2}(\omega)$ experimentally, cultured cortical cells expressing either $\mathrm{ChR} 2, \mathrm{ChR} 2 \mathrm{R}$, or $\mathrm{ChR} 2_{\mathrm{A}}$ were stimulated with spatially uniform blue light ( $465 \mathrm{~nm}$ at peak intensity) using a light emitting diode (LED), while somatic photocurrents were recorded using whole-cell patch clamp (Methods). To ensure that optical stimuli tracked intended stimulus waveforms, we developed a custom LED driver that used optical feedback to compensate for the static non-linearities and temperature dependence associated with the LED light source (Figures 3A-C; Methods).

To gain an initial confirmation of each variant's ability to relay continuously-varying photocurrents, we stimulated cells with swept frequency cosine ("chirp") stimuli (Figure 3D; Methods). Chirp inputs allow time and frequency characteristics of each variant to be read directly from the photocurrent time-series. Each variant displayed a characteristic decay in evoked current amplitude with increasing frequency, consistent with the model prediction. Additionally, the slightly increased midband amplitude of ChR2-evoked photocurrents provided indications of a bandpass effect. 


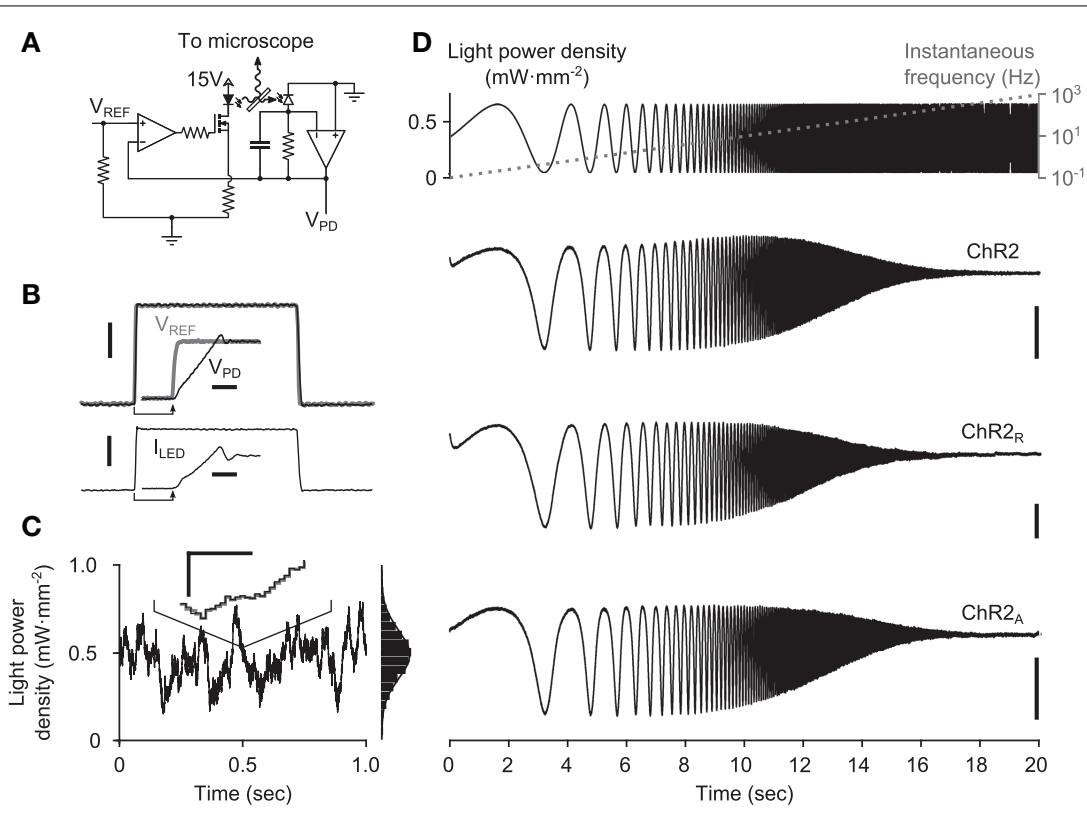

FIGURE 3 | Delivery of continuously-varying stimuli to neurons using ChR2. (A) Simplified schematic of the LED driver in optical feedback mode. The circuit uses an amplified photodiode to compensate for the non-linearities and temperature dependence of the LED, allowing arbitrary waveforms to be delivered to cells. (B) A 1-ms LED pulse, $V_{P D}$ (black), versus the reference voltage, $V_{R E F}$ (gray). The current sourced to the LED is shown in the lower plot. Scale bars, $1 \mathrm{~mW} \cdot \mathrm{mm}^{-2}$ (top) and $250 \mathrm{~mA}$ (bottom). Insets show the zoomed step onset with corresponding $5 \mu \mathrm{s}$ scale bars. (C) A computer generated
Gaussian stimulus (gray) signal and the recorded light waveform (black). The lines overlap almost perfectly, making the reference voltage (gray) difficult to see. An inset shows a zoomed portion of the sequence. Scale bars, $0.05 \mathrm{~mW} \cdot \mathrm{mm}^{-2}$ and $500 \mu \mathrm{s}$. An amplitude histogram of the sequence, with a best-fit Gaussian distribution, is shown to the right. (D) Responses to frequency chirp stimuli for each ChR2 variant tested. The top plot shows the stimulus waveform (black) along with the instantaneous frequency profile (gray) and bottom plots show evoked current waveforms. Scale bars, $100 \mathrm{pA}$.
Because the full dynamics of ChR2 are time-variant, the estimated frequency response (Equation 22; Methods) will vary depending on the stimulus choice. Our goal was to use ChR2 to deliver stimuli that mimic in vivo-like synaptic bombardment. Therefore, we used optical stimuli consisting of 10-s realizations of a filtered Gaussian noise signal (time constant: $\tau_{s}=50 \mathrm{~ms}$, mean \pm standard deviation: $\mu_{s}=0.4 \pm \sigma_{s}=0.08 \mathrm{~mW} \cdot \mathrm{mm}^{-2}$; Methods). We chose stimuli with these parameters because they evoked membrane voltage waveforms with similar amplitude and frequency characteristics to those obtained from in vivo recordings of sensory cortical neurons in the high-conductance state (Supplementary Figure S1) (Destexhe et al., 2003).

We measured the empirical frequency response function to the Gaussian noise stimulus, $\hat{F}_{\mathrm{ChR} 2}(\omega)$, of cells expressing ChR2 ( $n=9$ cells $), C h R 2_{\mathrm{R}}(n=4$ cells $)$, or $\mathrm{ChR}_{\mathrm{A}} \quad(n=6$ cells $)$ (Methods; Equations 21-24). We then compared the empirical amplitude responses for each variant, $\left|\hat{F}_{\mathrm{ChR} 2}(\omega)\right|$, with their theoretical counterparts. We observed good agreement between the empirically derived and predicted amplitude response functions, although some differences exist (Figure 1D). For instance, both $\left|\hat{F}_{\mathrm{ChR} 2 \mathrm{R}}(\omega)\right|$ and $\left|\hat{F}_{\mathrm{ChR} 2}(\omega)\right|$ have small downward deviations from the predicted response at $\sim 5 \mathrm{~Hz}$, which is more pronounced for ChR2. Additionally, the predicted frequency response tends to slightly overestimate the measured gain at frequencies above $100 \mathrm{~Hz}$. Because stimuli were spatially homogeneous and applied over the extent of the dentritic arbor, the lower-than-predicted response to high frequency stimuli may result from passive dendritic filtering of evoked currents.

To examine how the choice of stimulus waveform might change the channels' response properties, we recalculated the amplitude response function using photocurrents recorded in response to chirp stimuli (Supplementary Figure S2). Since chirp stimuli are sinusoidal, they result in a U-shaped amplitude distribution that emphasizes extreme stimulus amplitudes (0.05 and $0.65 \mathrm{~mW} \cdot \mathrm{mm}^{-2}$; Supplementary Figure S3). Because of large sinusoidal amplitude relative to the steady state light level $\left(\delta \phi / \phi_{0} \approx 1\right)$ and the overabundance of extreme values, chirp stimuli were less capable of meeting the condition $\mid(i \omega+$ $\left.\epsilon \phi_{0}\right) \mid \gg \epsilon \delta \phi$, which ensures agreement between the response predicted by $F_{\mathrm{ChR} 2}(\omega)$ and complete model solutions. Consequently, the amplitude response function estimated from chirp inputs deviates from the analytical amplitude response (Equation 19). As predicted using the model, these deviations primarily affect low frequencies $f \leq 5 \mathrm{~Hz}$ and are most prominent in wild-type ChR2 (Methods section "Robustness of the frequency response function"; Figure 2).

Despite these imperfections, both our theoretical and our empirical results indicate that all three channel types are capable of transmitting fluctuating current stimuli to populations of cells in a physiologically relevant frequency range (up to $\sim 100 \mathrm{~Hz}$ ). Furthermore, because the model provides a tractable description of channel dynamics, it serves as a useful tool for predicting the 
bandwidth and resonance of new channels based on measurable physiological parameters.

\section{RELIABILITY OF CONTINUOUSLY-VARYING ChR2-EVOKED CURRENTS}

In order for continuously-varying photostimulation to be useful in experimental settings, evoked current waveforms must be highly repeatable. Therefore, we measured the reliability of photocurrent waveforms across trials. As expected, evoked photocurrents looked like smoothed versions of the stimulus signal due to the low-pass effect of ChR2's amplitude response function (Figure 4). Evoked current waveforms were remarkably stable across trials. There there was no systematic change in the amplitude of evoked currents during repeated applications of a stimulus waveform (Figures 4A,C).

We next examined the distribution of evoked current amplitudes across cells (Figure 4C). The average standard deviation of photocurrents evoked by ChR2 was $26.7 \mathrm{pA}$. ChR2 $\mathrm{R}$ and $\mathrm{ChR} 2 \mathrm{~A}$ delivered only slightly larger current fluctuations than ChR2, with a mean standard deviations of $32.0 \mathrm{pA}$ and $31.4 \mathrm{pA}$, respectively. The similarity in evoked current amplitudes between ChR2 and the engineered variants is likely due to ChR2's resonance, which makes the channel most sensitive to fluctuating stimuli with power in the $10 \mathrm{~Hz}$ range, as opposed to steady state inputs.

Finally, to determine the reliability of evoked currents across cells, we calculated the normalized cross-correlation function, $c_{s, I_{i}}$, between the light power density, $s(t)$ and photocurrents
$I_{i}(t)$ for each cell, $i$, and across cells, $c_{I_{i}, I_{j}}$. For ChR2, the median peak value of $c_{I_{i}}, I_{j}$ was 0.96 , indicating strong correlations between evoked currents in different cells (Figure 4D). The median peak value of $c_{s, I_{i}}$ was 0.92 , indicating strong correlations between evoked currents and the stimulus waveform (Figure 4E). Additionally, the similarity in shape between $c_{I_{i}, I_{j}}$ and the autocorrelation of the stimulation process, $c_{s, s}$, indicates that temporal features of the stimulus were accurately converted into photocurrents, as predicted by the passband of the frequency response function (Figure 1).

\section{DISCUSSION}

Optogenetic methods offer genetic specificity, elimination of electrical recording artifacts, and increasingly specialized functionality (Berndt et al., 2011; Mattis et al., 2011). Because of these advantages, optogenetic methods are often used for direct manipulation of neuronal subpopulations in order to understand their function (Cardin et al., 2009; Sohal et al., 2009). Typically, pulsed optical stimuli are used to elicit neural responses. However, for studies that seek to understand information transmission in neural circuits, continuously modulated photocurrents that mimic synaptic bombardment offer several advantages over pulsed stimuli. Continuously modulated photocurrents provide highly controlled subthreshold inputs, while leaving the decision of when and how often to spike to individual neurons. Therefore, the spikes recorded from the network response to such continuously

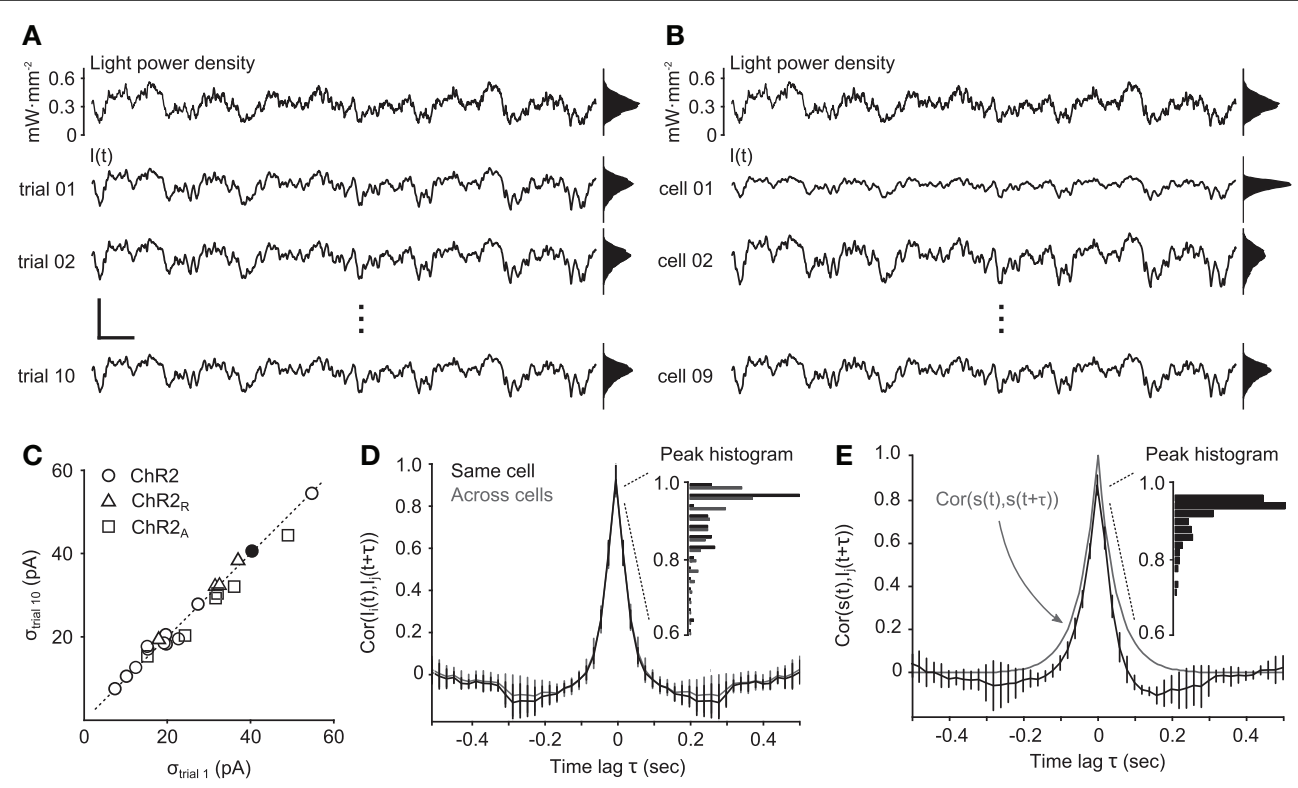

FIGURE 4 | Reliability of continuously-varying neuronal photostimulation. (A) Intracellular currents from a single cell during Gaussian stimuli. The top trace is a portion of a 10 -s Gaussian stimulus sequence. The bottom three traces show the intracellular currents recorded during different presentations of the same stimulus waveform. Scale bars, $200 \mathrm{pA}$ and $200 \mathrm{~ms}$. Scale bars apply to all time series traces in the figure. (B) The same stimulus waveform used in (A), and the corresponding evoked responses from different cells. (C) The standard deviation of the photocurrent induced on the first trial of stimulation versus on the last trial. The dotted line is identity. Points near the identity line indicate that there was little or no decrease in stimulus efficacy across trials. The filled dot corresponds to the cell in (A). (D) Normalized cross-correlation functions of photocurrents between neurons (gray) or autocorrelation function of photocurrents within the same neuron (black). The inset shows a histogram of peak correlation coefficients. (E) Normalized cross-correlation function between the stimulation process $s(t)$ and recorded photocurrents. The gray line is the autocorrelation function of the stimulation process. The inset shows a histogram of peak correlation coefficients. Unless otherwise noted, data in this figure were obtained from cells expressing ChR2. 
modulated photocurrents carry the signatures of innate spike generation mechanisms as well as those of interneuronal connectivity and thereby offer the possibility of revealing underlying network encoding strategies.

Previous studies of ChR2's response function have provided mixed results for understanding the channel's ability to relay time varying input. A preliminary abstract on the ChIEF (Lin et al., 2009) variant's response dynamics in HEK cells described a lowpass rather than a band-pass response (Neef et al., 2011). On the other hand, characterizations of numerous ChR2 variants have focused on step or pulse stimuli (Gunaydin et al., 2010; Mattis et al., 2011). To the best of our knowledge, this study is the first to both theoretically and experimentally derive the ChR2 channel's linear and complete response function in three ChR2 variants.

In this study, we demonstrated the ability of ChR2 to evoke continuously modulated photocurrents in neurons in response to continuously modulated light signals. We used a three-state Markov model (Nagel et al., 2003) to derive an analytical frequency response function for ChR2 variants (Equation 4). We confirmed these model predictions experimentally and have shown that the model is sufficient to capture dynamical properties of ChR2 in neurons within a physiologically relevant frequency range. Additionally, we found that the passband of ChR2, ChR2 $\mathrm{R}$, and $\mathrm{ChR} 2 \mathrm{~A}$ are broad enough to support photocurrents that mimic the noisy synaptic input received by neurons in the high conductance state, in vivo (Destexhe et al., 2003) (Supplementary Figure S1).

Aside from channel bandwidth, we found that temporal characteristics of continuously-varying photocurrents were highly repeatable across trials and cells. This contrasted with the amplitude distributions of the light-evoked currents across cells, which were highly variable (Figure 4C). The variability of photocurrent amplitudes results from non-homogeneous expression levels across cells. Potentially, this variability in channel expression could be used to simulate natural sources of noise in neural circuits such as variability in the number of incoming projections (Dayan and Abbott, 2001), variable spiking thresholds (Azouz and Gray, 2000), and sodium channel noise (Jacobson et al., 2005).

Continuously-varying optical stimuli allow subthreshold conductance modulations that can be spatially and genetically targeted. The spatially uniform optical stimuli used in our study produced highly correlated photocurrents across cells (Figure 4D). These temporally locked photocurrents mimic the highly correlated state of subthreshold thalamic drive to sensory cortical neurons that share a receptive field (Lampl et al., 1999; Roy and Alloway, 2001; Okun and Lampl, 2008). However, the incorporation of spatial light modulation would open the door to more complex experimental questions. For instance, spatial modulation of continuously-varying stimuli would allow control over the degree of synchronization between subthreshold currents across cells (Reutsky-Gefen et al., 2013). Additionally, spatial light modulation could be used to isolate continuously-varying input to particular regions of individual neurons (Grossman et al., 2010). For instance, by targeting the soma and axon-hillock, any low-pass effect resulting from the integrative properties of the dendritic arbor might be avoided. Conversely, targeting dendrites might provide more biophysically realistic input compared to stimuli covering the entire cell. However, even without spatial control of stimuli, we have shown that spatially homogeneous stimuli provide a wide bandwidth to deliver complex stimulus waveforms to populations of cells. Additionally, spatially homogeneous continuously-varying stimulation has the added benefit that it can be readily incorporated into existing experimental setups that use multi-mode optical fiber to deliver light in vivo.

Finally, we showed that ChR2's frequency response function supports a resonance. The degree of resonance is dependent on the values of free model parameters, which change for different ChR2 variants and stimulus signals. This finding is especially relevant for studies that use ChR2 to examine the frequencydependence of neural circuitry (Cardin et al., 2009), since it is important to separate the intrinsic dynamics of ChR2 from those that belong to the network under study. We found the most pronounced resonance for ChR2 with a natural frequency of $\sim 6-$ $10 \mathrm{~Hz}$. ChR2 was cloned from the green algae Chlamydomonas reinhardtii. Interestingly, the algae's phototaxic flagellar movement is tuned to the resonant frequency band of ChR2 (Josef et al., 2006), indicating a potential behavioral significance of ChR2's bandpass effect for algae in their natural environment.

ChR2's amplitude response function indicates that the sum of channel recovery and desensitization transition rates determines its frequency cut-off. Therefore, opsins with faster transition rates will allow a broader passband for time varying inputs. As new optogenetic tools are discovered and existing ones improved, their increased bandwidth may eventually offer an artificial, optical neural communication channel that actually exceeds the bandwidth of natural sensory organs. This would have tremendous implications for how neural computation and processing are studied and for the advancement of brain-machine interfaces. For the purposes of continuously-varying photostimulation with existing tools, we found that the $\mathrm{ChR} 2 \mathrm{~A}$ variant offered the widest dynamic range, did not display voltage-dependent channel kinetics, and exhibited only a mild resonance. This makes it a good choice for delivering continuously-varying stimuli to populations of cells embedded within functioning neural circuits.

ChR2 was derived from microbes that use it for optical sensation in natural environments. It is therefore not surprising that the channel is excellent at conveying continuously-varying input signals. Using channelrhodopsins as a means for delivering repeatable, continuously-varying stimuli to genetically defined populations of cells will be a powerful method for probing the dynamics of neural circuits and modulating their activity to provide artificial sensation.

\section{METHODS}

\section{CULTURING METHODS}

Our culturing methods are described in detail elsewhere (Hales et al., 2010). All experiments were carried out in accordance with the USA Public Health Service's Policy on Humane Care and Use of Laboratory Animals and the Guide for the Care and Use of Laboratory Animals using a protocol approved by the Georgia Tech IACUC. Timed-pregnant female rats were anesthetized with inhaled isoflurane and killed by decapitation. Whole brains were excised from embryonic day 18 (E18) rats. Cortical 
tissue was digested in a solution of $20 \mathrm{U} \cdot \mathrm{ml}^{-1}$ papain (Roche, Penzberg, Germany). Following enzymatic digestion, cells were mechanically dissociated using 3-5 trituration passes through a p1000 pipette tip. The resulting cell suspension was filtered through with a $40 \mu \mathrm{m}$ cell strainer and then centrifuged at $200 \cdot \mathrm{g}$ to remove large and small debris, respectively. The cell pellet was diluted to 2500 cells $\mu \mathrm{L}^{-1}$. Approximately 50,000 cells in a $20 \mu \mathrm{L}$ drop were plated at onto a $\sim 2 \mathrm{~mm}$ diameter area on glass bottom petri dishes, resulting in $\sim 16,000$ cells $\cdot \mathrm{mm}^{2}$ on the culturing surface. $0.75 \mathrm{~mL}$ of the culturing medium was exchanged every 3 days, for each culture. Cultures dishes were sealed with a Teflon membrane (Potter and DeMarse, 2001) and stored in an incubator regulated to $35^{\circ} \mathrm{C}, 5 \% \mathrm{CO}_{2}, 65 \%$ relative humidity.

\section{ChR2 EXPRESSION SYSTEM}

AAV2-CaMKll $\alpha:$ :ChR2-mCherry at $4 \cdot 10^{12}$ c.f.u. $\cdot \mathrm{ml}^{-1}$ was produced by the Kaplitt lab (Cornell University) using plasmid DNA for CaMKII $\alpha:$ ChR2-mCherry obtained from the K. Deisseroth (Standford University). AAV2-CaMKll $\alpha:$ hChR2(H134R)mCherry at $4 \cdot 10^{12}$ c.f.u. $\cdot \mathrm{ml}^{-1}$ was produced by the University of North Carolina at Chapel Hill Vector Core. AAV9CaMKll $\alpha::$ hChR2(E123A/H134R)-eYFP at $4 \cdot 10^{12}$ c.f.u. $\mathrm{ml}^{-1}$ was produced by the University of Pennsylvania Vector Core. At $1-5$ days in vitro (DIV), viral aliquots were diluted to $1 \cdot 10^{12}$ c.f.u. $\cdot \mathrm{ml}^{-1}$ using culturing medium. $1 \mu \mathrm{L}$ of diluted viral solution was added to $1 \mathrm{~mL}$ culturing medium for a final infection concentration of $1 \cdot 10^{9} \mathrm{c}$.f.u. $\cdot \mathrm{ml}^{-1}$. Cultures were then incubated for 3 days before the culturing medium was exchanged. The fluorescent signal of the reporter protein was monitored for several days post infection to ensure channel expression. All experiments were carried out at 3-4 weeks in vitro.

\section{INTRACELLULAR RECORDINGS}

Whole-cell voltage-clamp recordings were conducted on pyramidal neurons expressing the mCherry $(\mathrm{ChR} 2 \mathrm{R}$ and $\mathrm{ChR} 2)$ or eYFP $\left(C h R 2_{A}\right)$ reporter protein. Recordings were performed in a continuous perfusion of artificial cerebrospinal fluid (aSCF) bubbled with $95 \% \mathrm{O}_{2}$ and $5 \% \mathrm{CO}_{2}$ to maintain a $\mathrm{pH}$ of 7.4. The aSCF solution contained (in $\mathrm{mM}$ ) $126 \mathrm{NaCl}, 3 \mathrm{KCl}, 2$ $\mathrm{CaCl}_{2}, 1 \mathrm{NaH}_{2} \mathrm{PO}_{4}, 25 \mathrm{NaHCO}_{3}, 1.5 \mathrm{MgSO}_{4}$, and $25 \mathrm{D}$-glucose. The temperature of the extracellular medium was regulated to $35^{\circ} \mathrm{C}$ using an inline heater (Warner Instruments, Hamden, CT). $1.5 \mathrm{~mm}$ outer diameter, $1.1 \mathrm{~mm}$ inner diameter borosilicate glass capillaries (Sutter Instruments, Novato, CA) were pulled into patch pipettes and filled with a solution containing (in $\mathrm{mM}$ ) $100 \mathrm{~K}$-gluconate, $30 \mathrm{KCl}, 3 \mathrm{ATP}, 2 \mathrm{MgSO}_{4}, 0.5$ ethylene glycol tetraacetic acid and 10 HEPES adjusted to $\mathrm{pH} 7.4$ using $0.1 \mathrm{M}$ $\mathrm{KOH}$. Filled pipettes had resistances of 4-8 M $\Omega$. Voltage clamp recordings were performed using HEKA EPC8 amplifier and PatchMaster control software in whole-cell mode. Cells were held at $-70 \mathrm{mV}$ and membrane current measurements were amplified and low-pass filtered at $3 \mathrm{kHz}$ before being digitized at $20 \mathrm{kHz}$ and streamed to disk. Access resistance and seal resistance were monitored between stimulation protocols. Current clamp recordings were performed in "fast" mode using the same filter setting as voltage clamp. All experiments were performed in the presence of
$40 \mu \mathrm{M}$ 6-cyano-7-nitroquinoxaline-2,3-dione (CNQX), $50 \mu \mathrm{M}$ (2R)-amino-5-phosphonovaleric acid (AP5), $20 \mu \mathrm{M}$ bicuculline in order to prevent synaptic transmission. Whole-cell recordings were analyzed offline in MATLAB (The MathWorks, Natick, MA).

\section{OPTICAL STIMULATION}

A 10-watt (electrical power) light emitting diode (LED) was used for optical stimulation, with peak emission wavelength of $465 \mathrm{~nm}$ and $\sim 20 \mathrm{~nm}$ full width at half maximum intensity (LZ400B200, LEDEngin, San Jose, CA). To deliver optical stimuli to cultured neurons, the LED was focused into the epi-illumination port of an E600FN upright microscope (Nikon Corporation, Tokyo, Japan) and passed through a $40 \mathrm{X}$ objective lens. The light power produced by LEDs is affected by their temperature. Additionally, the relationship between forward diode current and irradiance is a static non-linearity. To compensate for these factors and deliver distortion-free optical stimuli, we designed a precision current source with integrated optical-feedback to drive our LED (Figure 3A). This circuit measures the instantaneous optical power produced by the LED using an amplified photodiode. It then adjusts the current sourced to the LED such that the optical power measurement matches a reference voltage supplied by a digital to analog converter (DAC; LIH 1600, HEKA Electronik, Lambrecht/Pfalz, Germany). The circuit can precisely modulate the LED brightness over a bandwidth of $50 \mathrm{kHz}$ (Figures 3B,C). A full design specification for the device is available online (https://potterlab.gatech.edu/newman/ wiki).

\section{DERIVATION OF ChR2's FREQUENCY RESPONSE}

The differential equations governing the Markov model, (Equations 1-3), are non-homogeneous with continuouslyvarying coefficients. For this reason, the frequency response function does not provide a full description of the model's dynamics. However, it serves as a useful simplification for describing ChR2's bandpass characteristics within local regions of optical intensity (Figure 1B). The full time-variant dynamics are not analytically solvable and required numerical simulations of response trajectories (Figure 2).

ChR2's frequency response function, $F_{\mathrm{ChR} 2}(\omega)$, can be obtained by considering the channels' response to a small sinusoidal light signal with a constant light level $\phi_{0}$,

$$
\phi(t)=\phi_{0}+\delta \phi \exp (j \omega t)
$$

where $\omega=2 \pi f$ and $f$ is the frequency of the sinusoid in $\mathrm{Hz}$ and $j=\sqrt{-1}$. The first order response dynamics of the open and closed states can then be described by a constant offset and periodic component,

$$
\begin{aligned}
& O(t)=O_{0}+\delta O \exp (j \omega t) \\
& D(t)=D_{0}+\delta D \exp (j \omega t)
\end{aligned}
$$

Within a local region of optical intensities, $\phi_{0} \pm \delta \phi$, changes in the open state, $\delta O$, or the desensitized state, $\delta D$, are proportional to changes in optical input, $\epsilon \delta \phi$. The proportionality factors for the open and desensitized states are the frequency response 
functions $F_{\mathrm{ChR} 2}(\omega)$ and $G_{\mathrm{ChR} 2}(\omega)$, respectively,

$$
\begin{aligned}
& \delta O=\epsilon \delta \phi F_{\mathrm{ChR} 2}(\omega) \\
& \delta D=\epsilon \delta \phi G_{\mathrm{ChR} 2}(\omega) .
\end{aligned}
$$

Differentiating Equations 9 and 10 and inserting the result into Equations 1 and 2 leads to

$$
\begin{aligned}
j \omega \delta O \exp (j \omega t)=\left[\epsilon \phi_{0}\left(1-O_{0}-D_{0}\right)\right]+\left[\epsilon \delta \phi\left(1-O_{0}-D_{0}\right)\right. \\
\\
\left.+\epsilon \phi_{0}(\delta O-\delta D)-\Gamma_{d} \delta O\right] \exp (j \omega t)+\mathcal{O}(2), \\
j \omega \delta D \exp (j \omega t)=\left[\Gamma_{d} O_{0}-\Gamma_{r} D_{0}\right]+\left[\Gamma_{d} \delta O-\Gamma_{r} \delta D\right] \exp (j \omega t) .
\end{aligned}
$$

By dropping all but the first-order terms of Equations 13 and 14 (meaning those terms proportional to $\exp (j \omega t)$ ), and removing the common factor $\exp (j \omega t)$, changes in the open and desensitized states due to changes in light power are given by

$$
\begin{aligned}
& j \omega \delta O=\epsilon \delta \phi\left(1-O_{0}-D_{0}\right)+\left(\epsilon \phi_{0}-\Gamma_{d}\right) \delta O-\epsilon \phi_{0} \delta D \\
& j \omega \delta D=\Gamma_{d} \delta O-\Gamma_{r} \delta D
\end{aligned}
$$

where $\left(1-O_{0}-D_{0}\right)=C_{0}$ is the steady-state probability of the channel being closed. Performing the necessary algebra to solve for $\delta O$ results in

$\delta O=\epsilon \delta \phi\left[\frac{C_{0}\left(j \omega+\Gamma_{r}\right)}{-\omega^{2}+j \omega\left(\Gamma_{r}+\epsilon \phi_{0}+\Gamma d\right)+\epsilon \phi_{0} \Gamma_{r}+\epsilon \phi_{0} \Gamma_{d}+\Gamma_{r} \Gamma_{d}}\right]$.

Finally, referencing Equation 11, ChR2's frequency response function for a local region of light intensities is calculated by dividing the left hand side of Equation 17 by $\epsilon \delta \phi$,

$$
\begin{aligned}
F_{\mathrm{ChR} 2}(\omega) & =\frac{\delta O}{\epsilon \delta \phi} \\
& =\frac{C_{0}\left(j \omega+\Gamma_{r}\right)}{-\omega^{2}+j \omega\left(\Gamma_{r}+\epsilon \phi_{0}+\Gamma_{d}\right)+\epsilon \phi_{0} \Gamma_{r}+\epsilon \phi_{0} \Gamma_{d}+\Gamma_{r} \Gamma_{d}}
\end{aligned}
$$

and the amplitude response is then given by

$$
\left|F_{\mathrm{ChR} 2}\right|=\frac{C_{0} \sqrt{\omega^{2}+\Gamma_{r}^{2}}}{\sqrt{\left(-\omega^{2}+\epsilon \phi_{0} \Gamma_{r}+\epsilon \phi_{0} \Gamma_{d}+\Gamma_{r} \Gamma_{d}\right)^{2}+\left(\omega\left(\Gamma_{r}+\epsilon \phi_{0}+\Gamma_{d}\right)\right)^{2}}} .
$$

In the high frequency limit, Equation 4 reduces to $\frac{C_{0}(j \omega)}{-\omega^{2}+j \omega\left(\Gamma_{r}+\epsilon \phi_{0}+\Gamma_{d}\right)} \propto \frac{C_{0}}{j \omega /\left(\Gamma_{r}+\epsilon \phi_{0}+\Gamma_{d}\right)+1}$.

EXPERIMENTAL VERIFICATION OF FREOUENCY RESPONSE FUNCTIONS To estimate the frequency response of $\operatorname{ChR} 2, \hat{F}_{\mathrm{ChR} 2}(\omega)$, we delivered optical stimuli, $s(t)$, consisting of $T=10 \mathrm{~s}$ realizations of a Gaussian (Ornstein-Uhlenbeck) noise process while recording evoked intracellular currents, $I_{i}(t)$, within a single cell, $i$. $s(t)$ was generated according to

$s\left(t_{n+1}\right)=\mu_{s}+s\left(t_{n}\right) \exp \left(-d t / \tau_{s}\right)+\sigma_{s} \xi\left(t_{n}\right) \sqrt{1-\exp \left(-2 d t / \tau_{s}\right)}$, where $s\left(t_{1}\right)=0 \mathrm{~mW} \cdot \mathrm{mm}^{-2}, \mu_{s} \simeq 0.35 \mathrm{~mW} \cdot \mathrm{mm}^{-2}$, and $\sigma_{s} \simeq$ $0.08 \mathrm{~mW} \cdot \mathrm{mm}^{-2}$ are the initial condition, mean, and standard deviation of the process, respectively. $\tau_{s}=50 \mathrm{~ms}$ is the correlation time of $s(t), d t=40 \mu \mathrm{s}$ is the DAC update period, and $\xi\left(t_{n}\right)$ is a random variable drawn from the standard normal distribution. Each cell was exposed to a single, repeated realization of $s(t)$ for $k=10$ trials. The first $500 \mathrm{~ms}$ of each trial was ignored to remove the non-stationary effects of the stimulator turning on. The recorded intracellular currents were averaged across trials,

$$
\left\langle I_{i}\right\rangle=\frac{1}{10} \sum_{k=1}^{10} I_{i, k}(t)
$$

to remove trial-to-trial noise. We then calculated the empirical frequency response function for each cell,

$$
\hat{F}_{\mathrm{ChR} 2, i}(\omega)=\frac{S_{s\left\langle I_{i}\right\rangle}}{S_{s s}}
$$

where $S_{s s}$ is the power spectrum of $s(t)$ and $S_{s\left\langle I_{i}\right\rangle}$ is the cross spectrum of $\left\langle I_{i}\right\rangle$ and $s(t)$. $S_{s s}$ and $S_{s\left\langle I_{i}\right\rangle}$ are defined as the Fourier transforms of the corresponding correlation function,

$$
\begin{aligned}
c_{s s}(\tau) & =\int_{-T}^{T} s(t) s(t+\tau) \mathrm{d} \tau \\
c_{s\left\langle I_{i}\right\rangle}(\tau) & =\int_{-T}^{T} s(t) I(t+\tau) \mathrm{d} \tau .
\end{aligned}
$$

Finally, we averaged $\hat{F}_{\mathrm{ChR} 2, i}(\omega)$ across cells to obtain the empirical frequency response for each construct, $\hat{F}_{\mathrm{ChR} 2}(\omega)$. To improve our estimate of the power spectra, we followed the procedure introduced in Higgs and Spain (2009) and used a frequency dependent window, equivalent to a Gaussian bandpass filter with standard deviation of $\sigma=2 \pi / \omega$ in the frequency domain. Spectra were evaluated at discrete increments, $\omega_{n}=2 \pi 10^{n}, n=$ $0.1,0.2, \ldots, 3$. Model parameters were obtained for each ChR2 variant by fitting the predicted frequency response function in Equation 4 to the experimental estimate, Equation 22.

In addition to Gaussian stimuli, we used cosine frequency sweeps ("chirps") consisting of $T=20$ s sinusoidal sweeps of constant amplitude $a_{s}$ and exponentially increasing frequency from $f_{0}=0.1$ to $f_{T}=1000 \mathrm{~Hz}$. They were defined as

$$
s(t)=a_{s} \cos (2 \pi f(t))+a_{0}
$$

where

$$
f(t)=f_{0}\left(f_{T} / f_{0}\right)^{\frac{t}{T}}
$$

and $a_{s} \simeq 0.3 \mathrm{~mW} \cdot \mathrm{mm}^{-2}$ and $a_{0} \simeq 0.35 \mathrm{~mW} \cdot \mathrm{mm}^{-2}$. The empirical frequency response was then estimated directly from the intracellular current recordings according to

$$
\hat{F}_{\mathrm{ChR} 2, i}^{\mathrm{chirp}}(\omega)=\frac{\mathcal{F}\left\langle I_{i}\right\rangle}{\mathcal{F}\langle s\rangle},
$$

where $\mathcal{F}$ denotes the Fourier transform. 


\section{ACKNOWLEDGMENTS}

We thank L. Abbott, K. Miller, and S. Fusi for fruitful discussions. We thank M. LaPlaca for providing tissue and J. T. Shoemaker for performing tissue harvests. We thank N. Laxpati, and the Kaplan Lab for assistance with virus production. We thank P. Wenner for patch recording expertise. Tatjana Tchumatchenko was funded by Volkswagen Foundation and the Center for Theoretical Neuroscience, Columbia University. Experimental work was supported by NSF COPN grant 1238097 and NIH grant 1R01NS079757-01. Additionally, this work was supported by NSF GRFP Fellowship 08-593 to Jonathan P. Newman, NSF GRFP Fellowship 09-603 to Ming-fai Fong, and NSF IGERT Fellowship DGE-0333411 to Jonathan P. Newman and Ming-fai Fong.

\section{SUPPLEMENTARY MATERIAL}

The Supplementary Material for this article can be found online at: http://www.frontiersin.org/journal/10.3389/fncir.2013. 00184/abstract

\section{REFERENCES}

Arabzadeh, E., Petersen, R. S., and Diamond, M. E. (2003). Encoding of whisker vibration by rat barrel cortex neurons: implications for texture discrimination. J. Neurosci. 23, 9146-9154. Available online at: http://www.jneurosci.org/content/23/27/9146.full

Azouz, R., and Gray, C. M. (2000). Dynamic spike threshold reveals a mechanism for synaptic coincidence detection in cortical neurons in vivo. PNAS 97, 8110-8115. doi: 10.1073/pnas.130200797

Berndt, A., Schoenenberger, P., Mattis, J., Tye, K. M., Deisseroth, K., Hegemann, P., et al. (2011). High-efficiency channelrhodopsins for fast neuronal stimulation at low light levels. PNAS 108, 7595-7600. doi: 10.1073/pnas. 1017210108

Boyden, E. S., Zhang, F., Bamberg, E., Nagel, G., and Deisseroth, K. (2005). Millisecond-timescale, genetically targeted optical control of neural activity. Nat. Neurosci. 8, 1263-1268. doi: 10.1038/nn1525

Cardin, J., Carlen, M., Meletis, K., Knoblich, U., Zhang, F., Deisseroth, K., et al. (2009). Driving fast-spiking cells induces gamma rhythm and controls sensory responses. Nature 459, 663-667. doi: 10.1038/nature08002

Chichilnisky, E. J. (2001). A simple white noise analysis of neuronal light responses. Network 12, 199-213. doi: 10.1080/net.12.2.199.213

Dayan, P., and Abbott, L. F. (2001). Theoretical Neuroscience: Computational and Mathematical Modeling of Neural Systems. Cambridge, MA: The MIT Press. ISBN-13: 978-0262541855

Destexhe, A., Rudolph, M., and Pare, D. (2003). The high-conductance state of neocortical neurons in vivo. Nat. Rev. Neurosci. 4, 739-751. doi: 10.1038/nrn1289

Geffen, M. N., Broome, B. M., Laurent, G., and Meister, M. (2009). Neural encoding of rapidly fluctuating odors. Neuron 61, 570-586. doi: 10.1016/j.neuron.2009.01.021

Grossman, N., Poher, V., Grubb, M. S., Kennedy, G. T., Nikolic, K., McGovern, B., et al. (2010). Multi-site optical excitation using ChR2 and micro-LED array. J. Neural Eng. 7, 16004. doi: 10.1088/1741-2560/7/1/016004

Gunaydin, L. A., Yizhar, O., Berndt, A., Sohal, V. S., Deisseroth, K., and Hegemann, P. (2010). Ultrafast optogenetic control. Nat. Neurosci. 13, 387-392. doi: $10.1038 / \mathrm{nn} .2495$

Hales, C. M., Rolston, J. D., and Potter, S. M. (2010). How to culture, record and stimulate neuronal networks on micro-electrode arrays. J. Vis. Exp. 39:e2056. doi: $10.3791 / 2056$

Higgs, M. H., and Spain, W. J. (2009). Conditional bursting enhances resonant firing in neocortical layer 2-3 pyramidal neurons. J. Neurosci. 29, 1285-1299. doi: 10.1523/JNEUROSCI.3728-08.2009

Jacobson, G., Diba, K., Yaron-Jakoubovitch, A., Oz, Y., Koch, C., Segev, I., et al. (2005). Subthreshold voltage noise of rat neocortical pyramidal neurones. J. Physiol. 564.1, 145-160. doi: 10.1113/jphysiol.2004.080903

Josef, K., Saranak, J., and Foster, K. W. (2006). Linear systems analysis of the ciliary steering behavior associated with negative-phototaxis in chlamydomonas reinhardtii. Cell Motil. Cytoskeleton 63, 758-777. doi: 10.1002/cm.20158

Kayser, C., Logothetis, N. K., and Panzeri, S. (2010). Millisecond encoding precision of auditory cortex neurons. Proc. Natl.
Acad. Sci. U.S.A. 107, 16976-16981. doi: 10.1073/pnas.10126 56107

Lampl, I., Reichova, I., and Ferster, D. (1999). Synchronous membrane potential fluctuations in neurons of the cat visual cortex. Neuron 22, 361-374. doi: 10.1016/S0896-6273(00)81096-X

Lesica, N. A., Jin, J., Weng, C., Yeh, C.-I., Butts, D. A., Stanley, G. B., et al. (2007). Adaptation to stimulus contrast and correlations during natural visual stimulation. Neuron 55, 479-491. doi: 10.1016/j.neuron.2007.07.013

Lin, J. Y., Lin, M. Z., Steinbach, P., and Tsien, R. Y. (2009). Characterization of engineered channelrhodopsin variants with improved properties and kinetics. Biophys. J. 96, 1803-1814. doi: 10.1016/j.bpj.2008.11.034

Mainen, Z. F., and Sejnowski, T. J. (1995). Reliability of spike timing in neocortical neurons. Science 268, 1503-1506. doi: 10.1126/science.7770778

Mattis, J., Tye, K., Ferenczi, E. A., Ramakrishnan, C., O’Shea, D. J., Prakash, R., et al. (2011). Principles for applying optogenetic tools derived from direct comparative analysis of microbial opsins. Nat. Meth. 99, 2, 159-172. doi: 10.1038/nmeth.1808

Nagel, G., Brauner, M., Liewald, J. F., Adeishvili, N., Bamberg, E., and Gottschalk, A. (2005). Light activation of channelrhodopsin-2 in excitable cells of Caenorhabditis elegans triggers rapid behavioral responses. Curr. Biol. 15, 2279-2284. doi: 10.1016/j.cub.2005.11.032

Nagel, G., Szellas, T., Huhn, W., Kateriya, S., Adeishvili, N., Berthold, P., et al. (2003). Channelrhodopsin-2, a directly light-gated cation-selective membrane channel. PNAS 100, 13940-13945. doi: 10.1073/pnas.1936192100

Neef, A., EL Hady, A., Nagpal, J., Broeking, K., Afshar, G., Geisel, T., et al. (2011). "Continuous dynamic photostimulation-delivering defined, in-vivo-like fluctuating stimuli with channelrhodopsins," in Neuroscience Meeting Planner (Washington, DC: Society for Neuroscience), Program No. 225.05.

Okun, M., and Lampl, I. (2008). Instantaneous correlation of excitation and inhibition during ongoing and sensory-evoked activities. Nat. Neurosci. 11, 535-537. doi: $10.1038 / \mathrm{nn} .2105$

Potter, S. M., and DeMarse, T. B. (2001). A new approach to neural cell culture for long-term studies. J. Neurosci. Meth. 110, 17-24. doi: 10.1016/S01650270(01)00412-5

Reutsky-Gefen, I., Golan, L., Farah, N., Schejter, A., Tsur, Land Brosh, I., and Shoham, S. (2013). Holographic optogenetic stimulation of patterned neuronal activity for vision restoration. Nat. Commun. 4, 1509. doi: 10.1038/ncomm s2500

Roy, S. A., and Alloway, K. D. (2001). Coincidence detection or temporal integration? What the neurons in somatosensory cortex are doing. J. Neurosci. 21, 2462-2473. Available online at: http://www.jneurosci.org/content/ 21/7/2462.full

Sohal, V., Zhang, F., Yizhar, O., and Deisseroth, K. (2009). Parvalbumin neurons and gamma rhythms enhance cortical circuit performance. Nature 459, 698-702. doi: 10.1038/nature07991

Tchumatchenko, T., Malyshev, A., Wolf, F., and Volgushev, M. (2011). Ultra-fast population encoding by cortical neurons. J. Neurosci. 31, 12171-12179. doi: 10.1523/JNEUROSCI.2182-11.2011

Warland, D. K., Landolfa, M., Miller, J. P., and Bialek, W. (1992). Analysis and Modeling of Neural Systems. New York, NY: Springer. doi: 10.1007/978-1-46153560-7

Warland, D. K., Reinagel, P., and Meister, M. (1997). Decoding visual information from a population of retinal ganglion cells. J. Neurophysiol. 78, 2336-2350.

Conflict of Interest Statement: The authors declare that the research was conducted in the absence of any commercial or financial relationships that could be construed as a potential conflict of interest.

Received: 15 July 2013; paper pending published: 17 September 2013; accepted: 31 October 2013; published online: 06 December 2013.

Citation: Tchumatchenko T, Newman JP, Fong M-f and Potter SM (2013) Delivery of continuously-varying stimuli using channelrhodopsin-2. Front. Neural Circuits 7:184. doi: $10.3389 /$ fncir.2013.00184

This article was submitted to the journal Frontiers in Neural Circuits.

Copyright (c) 2013 Tchumatchenko, Newman, Fong and Potter. This is an openaccess article distributed under the terms of the Creative Commons Attribution License (CC BY). The use, distribution or reproduction in other forums is permitted, provided the original author(s) or licensor are credited and that the original publication in this journal is cited, in accordance with accepted academic practice. No use, distribution or reproduction is permitted which does not comply with these terms. 